\title{
Avaliação do alinhamento postural e extensibilidade muscular pela escala SAROMM em crianças e adolescentes com Paralisia Cerebral
}

\author{
Postural alignment and muscle extensibility assessment's by the SARROMM scale in children and \\ teenagers with Cerebral Palsy \\ Evaluación de la alineación postural y la extensión muscular mediante la escala SAROMM en niños \\ y adolescentes con Parálisis Cerebral
}

Recebido: 29/10/2021 | Revisado: 07/11/2021 | Aceito: 10/11/2021 | Publicado: 17/11/2021

\author{
José Lucas de Souza Lima \\ ORCID: https://orcid.org/0000-0002-7411-7303 \\ Universidade Católica de Pernambuco, Brasil \\ E-mail: joselucas0504@gmail.com \\ Amanda Santos Vidal de Negreiros \\ ORCID: https://orcid.org/0000-0002-2024-0277 \\ Universidade Católica de Pernambuco, Brasil \\ E-mail: amandavidaldenegreiros@gmail.com \\ Ana Karolina Pontes de Lima \\ ORCID: https://orcid.org/0000-0002-6972-1186 \\ Universidade Católica de Pernambuco, Brasil \\ E-mail: ana.karolina.pontes@gmail.com
}

\begin{abstract}
Resumo
Justificativa: A Paralisia Cerebral (PC) é caracterizada por uma lesão cerebral na fase de sua estruturação e maturação. Os indivíduos acometidos apresentam alterações que causam desajustes posturais gerando déficit na movimentação. A SAROMM é uma escala que avalia indivíduos com PC, identificando mudanças posturais, o alinhamento da coluna vertebral, a extensibilidade dos músculos e a amplitude de movimento. Objetivo: Avaliar o alinhamento postural e extensibilidade muscular de crianças e adolescentes com PC através da escala de SAROMM. Métodos: O estudo do tipo observacional de corte transversal, foi realizado em um ambiente virtual para a aplicação da escala de SAROMM, com tempo médio da avaliação entre 15 e 30 min. Resultados: A amostra final contou com 8 crianças, com predominância do sexo masculino $(62,5 \%)$ e a diplegia, o diagnóstico topográfico mais presente $(62,5 \%)$. Com relação ao alinhamento postural foi identificado que 37,5\% obtiveram o mínimo de 0 na pontuação, indicando a ausência de limitações de alinhamento com correção ativa. Na seção de extensibilidade muscular, por articulação, foi observado que o paciente VI obteve uma pontuação de 0 demonstrando não possuir alterações detectadas nessa avaliação, já o paciente IV exibiu o maior valor nas pontuações de membro inferior demonstrando mais comprometimento que os demais. Conclusão: As crianças avaliadas que possuíam baixas pontuações no escore apresentaram habilidades motoras voluntárias como sentar, andar, rolar e engatinhar em detrimento das que não possuem essas habilidades e obtiveram pontuações mais altas.
\end{abstract}

Palavras-chave: Postura; Encefalopatias; Jovens.

\begin{abstract}
Justification: Cerebral Palsy (CP) is characterized by a brain lesion in the phase of its structuring and maturation. Affected individuals present alterations that cause postural maladjustments, generating a deficit in movement. SAROMM is a scale that assesses individuals with $\mathrm{CP}$, identifying postural changes, spinal alignment, muscle extensibility and range of motion. Objective: To assess postural alignment and muscle extensibility in children and adolescents with CP using the SAROMM scale. Methods: The observational cross-sectional study was carried out in a virtual environment for the application of the SAROMM scale, with an average evaluation time between 15 and 30 min. Results: The final sample consisted of 8 children, predominantly male (62.5\%) and diplegia, the most common topographic diagnosis $(62.5 \%)$. Regarding postural alignment, it was identified that $37.5 \%$ had a minimum score of 0 , indicating the absence of alignment limitations with active correction. In the section on muscle extensibility, by joint, it was observed that patient VI obtained a score of 0 , demonstrating no changes detected in this assessment, while patient IV exhibited the highest value in lower limb scores, demonstrating more compromise than the others. Conclusion: The assessed children who had low scores in the score had voluntary motor skills such as sitting, walking, rolling and crawling at the expense of those who do not have these skills and obtained higher scores.
\end{abstract}

Keywords: Posture; Brain diseases; Adolescent. 


\begin{abstract}
Resumen
Justificación: La parálisis cerebral (PC) se caracteriza por una lesión cerebral en la fase de su estructuración y maduración. Los individuos afectados presentan alteraciones que provocan desajustes posturales, generando déficits en el movimiento. SAROMM es una escala que evalúa a las personas con parálisis cerebral, identificando cambios posturales, alineación espinal, extensibilidad muscular y rango de movimiento. Objetivo: Evaluar la alineación postural y la extensibilidad muscular en niños y adolescentes con PC mediante la escala SAROMM. Métodos: El estudio observacional transversal se realizó en un ambiente virtual para la aplicación de la escala SAROMM, con un tiempo promedio de evaluación entre 15 y $30 \mathrm{~min}$. Resultados: La muestra final estuvo conformada por 8 niños, predominantemente varones $(62,5 \%)$ y diplejía, el diagnóstico topográfico más común $(62,5 \%)$. En cuanto a la alineación postural, se identificó que el 37,5\% tenía una puntuación mínima de 0 , lo que indica la ausencia de limitaciones de alineación con la corrección activa. En el apartado de extensibilidad muscular, por articulación, se observó que el paciente VI obtuvo una puntuación de 0 , demostrando que no se detectaron cambios en esta valoración, mientras que el paciente IV presentó el mayor valor en las puntuaciones de miembros inferiores, demostrando mayor compromiso que los demás. Conclusión: Los niños evaluados que tenían puntuaciones bajas en la puntuación tenían habilidades motoras voluntarias como sentarse, caminar, rodar y gatear a expensas de los que no tenían estas habilidades y obtuvieron puntuaciones más altas.
\end{abstract}

Palabras clave: Postura; Encefalopatías; Adolescente.

\title{
1. Introdução
}

Resultante de uma lesão no sistema nervoso central, a Paralisia Cerebral (PC) ou encefalopatia crônica não progressiva da infância (ECI) é caracterizada por uma lesão cerebral na fase de sua estruturação e maturação. A PC é decorrente de acontecimentos pré, peri e pós-natal e pode ser causada por infecções, hipóxia, alterações genéticas, prematuridade e entre outros fatores. Esse distúrbio permanente e não progressivo pode afetar a percepção, sensações, cognição e comunicação e originar problemas osteomioarticulares (Hollung \&, 2019; Lopes \&, 2018; Mota, 2018; Lopes \& Pfeifer, 2014; Soares \&, 2015; Silva \&, 2017).

A PC ocorre em 1 entre cada 500 nascidos vivos, sendo um fator incapacitante ao longo da vida daqueles com esse distúrbio. Essa condição ocasiona uma série de problemas posturais, levando a comprometimento motores, que irão depender da área lesada. Contudo, a maioria dos estudos apontam que grande parte das crianças possuem comprometimento que influencia na sua independência funcional e autonomia, acarretando então, atraso no seu desenvolvimento psicomotor (Lopes \&, 2019; Lino \&, 2020; Park \& Johnston, 2006; Soares, et al., 2015; Gasch \& Traver, 2019; Silva, et al., 2017; Sousa Júnior \&, 2017).

Referente ao quadro clínico desses indivíduos, pode-se observar fraqueza muscular, rigidez muscular, alterações de tônus (hipertonia espástica ou hipotonia), déficit na marcha, movimentos involuntários, dificuldades na deglutição, problemas fonográficos e em alguns casos alterações cognitivas. A PC pode se apresentar nas formas quadriplégica espástica, diplégica espástica, hemiplégica espástica, discinética, atáxica e pode também exibir outras configurações não identificadas (Lopes, et al., 2018; Santos \&, 2019; Soares, et al., 2015; Silva, et al., 2017; Chappell \&, 2020).

Nessa disfunção os pacientes apresentam alterações tônicas e tróficas, que causam desajustes posturais gerando déficit na movimentação, o que pode levar essas crianças e adolescentes a desenvolver contraturas e deformidades periféricas. As principais alterações encontradas no nível da coluna vertebral são hipercifose, retificação da lordose e escoliose, que prejudica o desenvolvimento infantil e traz variados padrões de postura e marcha. Assim é imprescindível a avaliação postural do fisioterapeuta (Silva, et al., 2017; Park \& Johnston, 2016; Santos, et al., 2019; Santos \& Alves, 2019; Gasch \& Traver, 2019; Figueiredo \&, 2020).

A The Spinal Alignment and Range of Motion Measure (SAROMM) é uma escala criada para avaliar indivíduos com PC, identificando mudanças posturais, o alinhamento da coluna vertebral, a extensibilidade dos músculos e a amplitude de movimento de áreas como, quadril, ombro, joelho e extremidades. A escala foi criada para analisar e diagnosticar padrões de 
posturas e amplitude de movimento típicas ou atípicas, com a finalidade de guiar as decisões para o tratamento, objetivos e métodos terapêuticos (Santos \& Alves, 2019; Bartlett \& Purdie, 2005; Brasil, 2014; Lopes \& Pfeifer, 2014).

Não existem muitos trabalhos para avaliar o alinhamento postural e a extensibilidade muscular em crianças e adolescentes com PC. Dito isto, é importante o estudo através da escala de SAROMM, pois essa avaliação pode fornecer informações para o diagnóstico cinético funcional dessas crianças e adolescentes e um perfil clínico dessa população, auxiliando na elaboração de um planejamento terapêutico mais efetivo. O objetivo do artigo é avaliar o alinhamento postural e a extensibilidade muscular de crianças e adolescentes com PC através da escala de SAROMM.

\section{Metodologia}

O estudo está vinculado a Universidade Católica de Pernambuco, a Escola de saúde e ciências da vida e ao curso de Fisioterapia. Parte integrante do projeto de pesquisa "Fisioterapia nas disfunções do sistema nervoso central e periférico" e pertencente ao grupo de pesquisa "Fisioterapia baseada em evidências" sob responsabilidade da Profa. Ana Karolina Pontes de Lima.

O estudo do tipo observacional, quantitativo, de corte transversal, foi realizado com 8 crianças e adolescentes que apresentavam PC de ambos os gêneros. O tipo de seleção foi por conveniência, baseado no número de crianças e adolescentes que frequentavam os laboratórios. Foram incluídas crianças com idade entre 1 a 17 anos, com diagnóstico médico de PC, sendo excluídas crianças com malformação congênita não relacionada à PC e doenças cromossômicas.

O estudo foi realizado através da plataforma virtual Google Meet, que é um serviço de comunicação por vídeo desenvolvido pelo Google, da residência de cada participante. Foi realizado o contato com os pacientes, com explicações detalhadas sobre o projeto, a assinatura do Termo Livre e Esclarecido e do Termo de Assentimento Livre e Esclarecido e informado que a participação é voluntária, apresentando o direito de desistir a qualquer momento. Inicialmente, foi realizada uma análise dos prontuários das crianças e adolescentes que se enquadram na amostra, averiguando toda a história da gestação e da criança nos primeiros meses de vida.

Posteriormente, foi marcado um encontro no ambiente virtual utilizando o Meet e o Google forms para aplicar a escala The Spinal Alignment and Range of Motion Measure (SAROMM), que é destinada para pessoas com o diagnóstico de PC tendo um tempo médio de conclusão da avaliação entre 15 e 30 minutos dependendo dos problemas físicos e cognitivos, sendo divididos em "sem limitações" e "desvios graves", baseando-se na avaliação do alinhamento postural para definir em típica ou atípica. A escala é dividida em duas partes, a primeira sendo formada por quatro itens de avaliação da coluna vertebral e a segunda por onze itens, para a avaliação da amplitude de movimento e extensibilidade muscular, que deve ser testada bilateralmente. Essas informações possuem pontuações para cada item, sendo variado de zero a quatro pontos, onde zero indica que ocorre o alinhamento sem limitações e quatro que apresenta desvios graves na coluna vertebral ou limitação na amplitude de movimento ou extensibilidade muscular. No alinhamento postural será avaliada a coluna cervical, torácica, lombar e curvatura lateral, já na extensibilidade muscular será avaliado: extensão do quadril, flexão do quadril, abdução do quadril, adução do quadril, rotação externa e interna do quadril, extensão dos joelhos e ísquiotibiais. O resultado é definido de acordo com o total que é a soma dos valores obtidos, dividida pela quantidade de articulações avaliadas (Santos, et al., 2019).

Foi orientado aos participantes que para efetuar a escala é necessária uma superfície firme onde o indivíduo seja capaz de se sentar, com o quadril e joelhos flexionados em aproximadamente 90 graus e um piso, tablado, cama ou maca para testes na posição supina. $\mathrm{O}$ indivíduo sendo incapaz de se manter sentado no banco independentemente pode ser necessário duas pessoas para efetuar o teste. Definindo onde cada paciente se encaixa, é determinado se os indivíduos apresentam o alinhamento e a amplitude de movimento típica ou atípica pelos valores obtidos na avaliação (Santos, et al., 2019). 
A pesquisa seguiu as normas da resolução 466/12, que incorpora sob a ótica do indivíduo e das coletividades os quatro referenciais básicos da bioética: autonomia, não maleficência, beneficência e justiça, e visa assegurar os direitos e deveres que dizem respeito à comunidade científica, aos sujeitos da pesquisa e ao Estado. Com os valores da coleta de dados, foi construído um banco de dados utilizando o Programa Microsoft Excel versão 7.0. Os resultados referentes às variáveis de interesse do estudo foram analisados e expostos em tabelas e gráficos.

\section{Resultados e Discussão}

A amostra final do estudo contou com 8 crianças, sendo o sexo masculino o mais predominante (62,5\%), com uma média de idade de 9 anos. Em relação à escolaridade, a maioria das crianças cursavam o ensino fundamental (62,5\%), e apresentavam como diagnóstico topográfico a diplegia (62,5\%) (Tabela 1).

Tabela 1: Características sócio clínicas das crianças e adolescentes com PC ( $\mathrm{n}=8)$.

\begin{tabular}{ccccc}
\hline & SEXO & IDADE & ESCOLARIDADE & DIAGNÓSTICO TOPOGRÁFICO \\
\hline PCTE I & $\mathrm{M}$ & 5 ANOS & EDUCAÇÃO INFANTIL & Quadriplegia \\
PCTE II & $\mathrm{M}$ & 12 ANOS & EDUCAÇÃO FUNDAMENTAL & Diplegia \\
PCTE III & $\mathrm{M}$ & 10 ANOS & EDUCAÇÃO FUNDAMENTAL & Diplegia \\
PCTE IV & $\mathrm{F}$ & 10 ANOS & EDUCAÇÃO FUNDAMENTAL & Diplegia \\
PCTE V & $\mathrm{F}$ & 13 ANOS & EDUCAÇÃO FUNDAMENTAL & Diplegia \\
PCTE VI & $\mathrm{M}$ & 2 ANOS & EDUCAÇÃO INFANTIL & Hemiplegia \\
PCTE VII & $\mathrm{F}$ & 15 ANOS & EDUCAÇÃO FUNDAMENTAL & Quadriplegia \\
PCTE VIII & $\mathrm{M}$ & 5 ANOS & EDUCAÇÃO INFANTIL & Diplegia \\
\hline
\end{tabular}

Legenda: PCTE = Paciente; $\mathrm{M}=$ Masculino; $\mathrm{F}=$ Feminino. Fonte: Autores.

No presente estudo houve uma predominância do sexo masculino (62,5\%). Em contrapartida, Correia (2016), no trabalho que verificou os universos socioeconômico e demográfico da criança com PC, notaram o sexo feminino como o mais prevalente (52\%). Já Vieira e Chagas (2017), em seu artigo que objetivou avaliar o perfil funcional e sociodemográfico dos portadores de PC, encontraram uma predominância do sexo masculino (50,8\%), tal qual o estudo em questão. Em relação ao diagnóstico topográfico Vieira e Chagas (2017) verificaram uma porcentagem de 61,8\% para quadriplegia, um resultado divergente do atual estudo, que apresentou a diplegia como a mais comum. No que diz respeito a idade da amostra, a média foi de 9 anos.

Durante a avaliação do alinhamento postural foi observado a coluna cervical, coluna torácica, coluna lombar e curva lateral, com pontuações de 0 a 4 . Nas crianças e adolescentes avaliadas, 37,5\% obtiveram o mínimo de 0 na pontuação, indicando a ausência de limitações de alinhamento com correção ativa. Já o máximo apresentado de pontuação nos pacientes foi 3 (a limitação é estrutural, estática, não redutível e moderada tento alterações moderadas na coluna lombar e curva lateral). Já na coluna cervical e torácica, $50 \%$ da amostra apresentou a pontuação 2 (a limitação é estrutural, estática, não redutível e mínima). Nenhum paciente avaliado apresentou alterações severas em seus alinhamentos posturais. A média foi de 0,75 na coluna cervical, 1,125 na coluna torácica, 1,375 na coluna lombar e 1,875 na curva lateral (Tabela 2). 
Tabela 2: Alinhamento postural pela escala de SAROMM das crianças e adolescentes com PC $(\mathrm{n}=8)$.

\begin{tabular}{ccccc}
\hline & COLUNA CERVICAL & COLUNA TORÁCICA & COLUNA LOMBAR & CURVA LATERAL \\
\hline PCTE I & 1 & 2 & 2 & 3 \\
PCTE II & 0 & 0 & 0 & 1 \\
PCTE III & 1 & 2 & 2 & 3 \\
PCTE IV & 1 & 2 & 2 & 2 \\
PCTE V & 0 & 0 & 1 & 1 \\
PCTE VI & 0 & 0 & 0 & 0 \\
PCTE VII & 2 & 3 & 3 \\
PCTE VIII & 1 & 1 & 2 \\
Mínimo & 0 & 1 & 0 & 0 \\
Máximo & 2 & 0 & 3 & 3 \\
Média & 0,75 & 2 & 1,375 & 1,875 \\
Desvio padrão & 0,707106781 & 1,125 & 1,060660172 & 1,125991626 \\
\hline
\end{tabular}

Legenda: PCTE $=$ PACIENTE. Fonte: Autores.

Com base nos estudos utilizando a escala de SAROMM pode-se afirmar que essa escala vai fornecer um método de avaliação confiável, porém com algumas variações por terapeuta. Bartlett \& Purdie (2005) alegam que é um método confiável a ser utilizado em um ambiente comunitário por profissionais de reabilitação. Nesse mesmo pensamento Chen \& (2013), abordam que a escala possui uma validade razoavelmente boa para avaliação de crianças com PC, sendo colocado que é necessário ter um treinamento breve para cada avaliador para fidelizar os resultados, sendo um método simples, de boa eficácia e válido para estimar o alinhamento geral da coluna e a ADM em crianças com PC quando comparados com métodos de avaliação como a goniometria.

Com relação ao alinhamento postural foi identificado que a criança com a menor pontuação (PCTE VI $=0$ no alinhamento postural) além de possuir a menor idade, também apresentou habilidades voluntárias de correção de posturas. Dados também encontrados por Bartlett \& Purdie (2005), que afirmaram que os escore da SAROMM estão significativamente relacionados não somente as idades das crianças, mas também a suas atividades motoras grossas, no movimento iniciado voluntariamente. Corroborando com essa informação Jeffries \& (2019) sustentam que crianças mais novas e com essas habilidades voluntárias vão possuir uma pontuação menor comparadas àquelas que não possuem essas atividades de forma voluntária, isso se dá muito provavelmente pela habilidade de correção voluntária, diminuindo os riscos de alterações no alinhamento postural.

Na seção de extensibilidade muscular, por articulação, foi observado que o paciente VI obteve uma pontuação de 0 demonstrando não possuir alterações detectadas nessa avaliação, já o paciente IV exibiu o maior valor nas pontuações de membro inferior demonstrando mais comprometimento que os demais, obtendo o máximo de 34 no quadril, 12 no joelho e 8 no tornozelo (Tabela 3). 
Tabela 3: Extensibilidade pela escala de SAROMM das crianças e adolescentes com PC $(n=8)$.

\begin{tabular}{ccccc}
\hline & QUADRIL & JOELHO & TORNOZELO & MEMBROS \\
\hline PCTE I & 20 & 6 & 6 & 2 \\
PCTE II & 12 & 4 & 4 & 0 \\
PCTE III & 27 & 10 & 8 & 0 \\
PCTE IV & 34 & 12 & 8 & 0 \\
PCTE V & 24 & 8 & 8 & 0 \\
PCTE VI & 0 & 0 & 0 & 6 \\
PCTE VII & 24 & 12 & 8 & 2 \\
PCTE VIII & 30 & 12 & 8 & 0 \\
Mínimo & 0 & 0 & 0 & 6 \\
Máximo & 34 & 12 & 8 & 1,75 \\
Média & 21,375 & 8 & 6,25 & 2,251983253 \\
Desvio padrão & 10,86195852 & 4,40778532 & 2,915475947 & \\
\hline
\end{tabular}

Legenda: PCTE $=$ Paciente. Fonte: Autores.

Com relação a extensibilidade muscular foi encontrado nas crianças com maiores pontuações (PCTE VI=54) uma idade mais elevada (10 anos) e uma diminuição nas atividades motoras grossas (sentar, transferências, rolar, engatinhar e mobilidade) com uma maior ênfase nos membros inferiores. Resultados também observados por Bartlett \& Purdie (2005), que avaliaram a postura e flexibilidade para crianças com PC. Diferente do presente estudo, onde a maioria das crianças apresentavam movimentação voluntária, Jeffries et al. (2019), verificaram que crianças que não possuem movimentação voluntária podem apresentar uma diminuição na extensibilidade muscular tal qual foi observado. Os autores afirmam também que este para ser um ótimo método para avaliar pacientes que não cooperam e para crianças pequenas, sem interferência do cognitivo do avaliado.

No escore final, demonstrado na Tabela 4, tem-se os valores somados do alinhamento postural mais os valores da extensibilidade muscular, que variam de 0 a 104. Os valores mais próximos a 104 indicam maior comprometimento dos aspectos avaliados. Na avaliação apenas um paciente apresentou o valor mínimo (paciente VI) demonstrando nenhum comprometimento no alinhamento postural e na extensibilidade muscular. Já o paciente mais comprometido apresentou um escore de 61 (paciente IV). A amostra apresentou uma média de 42,5 no valor final da escala (Tabela 4).

Tabela 4: Escore total da escala de SAROMM das crianças e adolescentes com PC ( $\mathrm{n}=8)$.

\begin{tabular}{cccc}
\hline & ALINHAMENTO POSTURAL & EXTENSIBILIDADE MUSCULAR & SCORE TOTAL \\
\hline PCTE I & 8 & 34 & 42 \\
PCTE II & 1 & 20 & 21 \\
PCTE III & 8 & 49 & 57 \\
PCTE IV & 7 & 54 & 61 \\
PCTE V & 2 & 40 & 42 \\
PCTE VI & 0 & 0 & 0 \\
PCTE VII & 10 & 50 & 60 \\
PCTE VIII & 5 & 52 & 57 \\
Mínimo & 0 & 0 & 0 \\
Máximo & 10 & 54 & 61 \\
Média & 5,125 & 37,375 & 42,5 \\
Desvio & 3,720119046 & 18,90530916 & 21,83705893 \\
padrão & & & \\
\hline
\end{tabular}


Dentro das subescalas Alinhamento postural e Extensibilidade muscular foi possível observar, nas duas modalidades, que a idade e as habilidades motoras grossas voluntárias com ênfase no sentar, nas transferências, mobilidade e nas correções posturais tinham relação com o valor dentro do escore da escala. Bartlett e Purdie (2005) analisaram que o escore da SAROMM está significativamente relacionado não somente às idades das crianças, mas também a suas atividades motoras grossas, no movimento iniciado voluntariamente e na correção postural. Nesse mesmo sentindo, Chen et al. (2013) constataram que crianças mais novas e com essas habilidades voluntárias vão possuir uma pontuação menor comparadas àquelas que não realizam as atividades de forma voluntária.

Neste trabalho a amostra foi avaliada uma única vez de forma remota, não sendo submetidas a uma intervenção e nem a uma segunda avaliação, o que diverge dos trabalhos encontrados, os quais foram submetidos a uma comparação tanto de terapeutas diferentes quanto a momentos diferentes para observar se houve alguma alteração nos resultados. Bartlett e Purdie (2005) afirmam que a pontuação de confiabilidade de teste e reteste estão acima de 0,80 o que suporta o uso da pontuação das subescalas ou pontuação total da SAROMM. A confiabilidade usando este tipo de escala de classificação é considerada 'alta' e o uso de estimativa visual tem implicações práticas, pois apenas uma pessoa é necessária para estimar a amplitude de movimento de crianças ou adolescentes com PC, principalmente se utilizando do ambiente virtual para avaliar de forma remota, como foi observado neste trabalho.

\section{Considerações Finais}

Foi possível concluir que as crianças avaliadas que possuem uma baixa pontuação no escore apresentaram habilidades motoras voluntárias como sentar, andar, rolar e engatinhar em detrimento das que não possuam essas habilidades e obtiveram pontuações mais altas. Assim pode-se constatar que escala de SAROMM tem uma aplicabilidade prática, simples e válida para utilizar como parâmetros de avaliação em crianças e adolescentes com PC, pois fornece um feedback visual e numérico, e pode ser considerado um importante método de avaliação a ser implantado em outros estudos. Contudo, ainda é uma ferramenta pouco utilizada e abordada, tendo limitadas evidências na literatura como um instrumento de avaliação.

Desta forma, faz-se necessário uma continuidade nesse estudo, bem como novos que envolvam a utilização dessa escala como forma de avaliação desses pacientes, na forma de ensaios clínicos randomizados.

\section{Referências}

Bartlett, D., \& Purdie, B. (2005). Testing of the Spinal Alignment and Range of Motion Measure: a discriminative measure of posture and flexibility for children with cerebral palsy. Developmental Medicine \& Child Neurology, 47(11), 739.

Brasil. Ministério da Saúde. Ministério da Saúde. (2014) Diretrizes de Atenção à Pessoa com Paralisia Cerebral. Brasília: Ministério da Saúde Secretariaexecutiva Subsecretaria de Assuntos Administrativos Coordenação-geral de Documentação e Informação Coordenação de Gestão Editorial. 74 p.

Cervera Gasch, A., \& Martínez Traver, L. (2019). Calidad de vida en adultos institucionalizados con parálisis cerebral infantil. Enfermería Global, 19(1), 243262.

Chappell, A., Allison, G. T., Williams, G., Gibson, N., \& Morris, S. (2020). The effect of a running training intervention on ankle power generation in children and adolescents with cerebral palsy: A randomized controlled trial. Clinical Biomechanics, 76, 105024.

Chen, C.-L., Wu, K. P. H., Liu, W.-Y., Cheng, H.-Y. K., Shen, I.-H., \& Lin, K.-C. (2013). Validity and clinimetric properties of the Spinal Alignment and Range of Motion Measure in children with cerebral palsy. Developmental Medicine \& Child Neurology, n/a.

Correia, V. D., \& Salimene, A. C. d. M. (2016). Social profile of children and teens with cerebral palsy in a physical rehabilitation center. Acta Fisiátrica, 23(1).

Figueiredo, A. A., Lomazi, E. A., Montenegro, M. A., \& Bellomo-Brandão, M. A. (2020). Quality of life in caregivers of pediatric patients with cerebral palsy and gastrostomy tube feeding. Arquivos de gastroenterologia, 57, 3-7.

Hollung, S. J., Bakken, I. J., Vik, T., Lydersen, S., Wiik, R., Aaberg, K. M., \& Andersen, G. L. (2019). Comorbidities in cerebral palsy: a patient registry study. Developmental Medicine \& Child Neurology, 62(1), 97-103. 
Research, Society and Development, v. 10, n. 15, e54101522502, 2021

(CC BY 4.0) | ISSN 2525-3409 | DOI: http://dx.doi.org/10.33448/rsd-v10i15.22502

Jeffries, L. M., LaForme Fiss, A., Westcott McCoy, S., Bartlett, D., Avery, L., \& Hanna, S. (2019). Developmental Trajectories and Reference Percentiles for Range of Motion, Endurance, and Muscle Strength of Children With Cerebral Palsy. Physical Therapy, 99(3), 329-338.

Lino, T. B., Martinez, L. B. A., Boueri, I. Z., \& Lourenço, G. F. (2020). Efeitos do Uso de Recursos de Tecnologia Assistiva para Promover Independência em Atividades de Vida Diária para uma Criança com Paralisia Cerebral2. Revista Brasileira de Educação Especial, 26, 35-50.

Lopes, J., Prieto, A. V., Santos, J. A. T., Smaili, S. M., \& Gutierres Filho, P. J. B. (2019). Efetividade da equoterapia na marcha de crianças com paralisia cerebral: revisão sistemática de ensaios clínicos. Revista Brasileira de Neurologia, 55(1), 25-34.

Lopes, R. R., \& Pfeifer, L. I. (2014). Adaptação transcultural e estudo preliminar da escala Spinal Alignment and Range of Motion Measure - SAROMM [Dissertação]. Ribeirão Preto: Faculdade de Medicina de Ribeirão Preto.

Lopes, R. R. B., Mestriner, T., Costa, C. S. N., \& Pfeifer, L. I. (2018). Postural Impairment of Adults and Adolescents Cerebral Palsy Inpatients at the Retaguarda Hospital of Ribeirão Preto-SP. J Neurol Neurol Disord, 4(1), 101.

Mota, A. P., \& Pereira, J. S. (2006). Influência da fisioterapia nas alterações motoras em crianças com paralisia cerebral. Fisioter. Bras, $209-212$.

Park, T. S., \& Johnston, J. M. (2006). Surgical techniques of selective dorsal rhizotomy for spastic cerebral palsy. Neurosurgical focus, 21 (2), E7.

Santos, D. L. A. D., \& Alves, J. S. (2019). Tradução e adaptação cultural para o português do Brasil da escala de avaliação spinal alignment and range of motion measure-SAROMM.

Santos, R. A., Da-Silva, V. R., dos-Santos, J. P., \& Siqueira, A. N. Epidemiological and healthcare access profiles of children and adolescents with cerebral palsy in a municipality in the brazilian state of Espírito Santo.

Silva, E. M. D., Silva, T. A. D. S. D., Balk, R. D. S., Lopes, R. R., Santos, C. C., Lara, S., \& Graup, S. (2017). Avaliação do alinhamento postural e extensibilidade muscular pela escala SAROMM em crianças com paralisia cerebral após fisioterapia aquática. Fisioter. Bras, f-719.

Soares, M. D., Santos, J. K. V., Costa, F. A., \& Melo, L. P. (2015). Wii rehabili-tation and neurological physiotherapy: a systematic review. Wii reabilitação e fisioterapia neurológica: uma.

Sousa, R. R. D., Lima, P. D., Silva, J. N. D., \& Vaz, D. V. (2017). Effects of kinesiology taping in children with cerebral palsy: a systematic review. Fisioterapia em Movimento, 30, 373-382.

Vieira, J. L. R., \& Chagas, J. R. L. P. (2017). Perfil funcional e sócio demográfico dos pacientes com paralisia cerebral em reabilitação num centro de referência em salvador. Revista Brasileira de Neurologia e Psiquiatria, 21(2). 